\title{
Organisation du travail dans des élevages familiaux lait et viande sur un front pionnier amazonien au Brésil. Etude à partir de sept enquêtes "bilan travail "
}

\author{
N. Hostiou ${ }^{1 *}$ J.F. Tourrand ${ }^{2}$ J.B. Veiga ${ }^{2}$
}

\begin{abstract}
Mots-clés
Bovin de boucherie - Bovin laitier Organisation du travail - Conduite du bétail - Amazonie - Brésil.
\end{abstract}

\begin{abstract}
Résumé
Cette étude privilégie un regard nouveau sur les élevages laitiers familiaux en frontière agricole au Brésil : I'organisation technique du travail. Le travail apparaît être un facteur essentiel des conduites d'élevage et une contrainte aux transformations durables des exploitations. Cependant très peu de connaissances ont été produites jusqu'à ce jour. Pour rendre compte de l'organisation du travail sur une année, les temps de travaux ont été quantifiés et les collectifs de travail décrits dans sept fermes "lait-viande», à partir de la méthode « bilan travail ». Les mêmes activités d'astreinte avec le troupeau laitier ont été réalisées dans tous les élevages avec des variances quant aux volumes horaires : 1 h 45 à 6 h 30 par jour et par personne de la cellule de base. Les différences entre les fermes ont été liées à la composition de la cellule de base, à l'effectif de vaches traites et au mode de commercialisation du lait. Sur I'année, 17 à 328 journées ont été dédiées aux activités de saison, dont 17 à 176 jours sur les prairies cultivées. Une partie des activités sur les prairies (sarclage, implantation, clôtures) ont été déléguée à de l'aide familiale ou à des salariés temporaires. Trois stratégies d'éleveurs face au travail ont été identifiées, reposant sur la simplification des conduites ou l'organisation des collectifs de travail. L'étude conclut que le travail est un facteur à prendre en compte pour appuyer la transformation des pratiques des éleveurs et la durabilité des élevages familiaux amazoniens. Les futures actions de recherche-développement en Amazonie permettront de produire des connaissances pour identifier les voies d'amélioration possibles.
\end{abstract}

\section{INTRODUCTION}

En frontière agricole amazonienne, l'élevage bovin tient une place croissante dans les systèmes de production familiaux (8). Depuis ces dernières années, une activité laitière commerciale se met en place dans ces fermes (14). Considérée comme un facteur de consolidation des exploitations familiales pour des raisons économiques, sociales et organisationnelles (15), elle fait l'objet d'actions de recherche-développement pour appuyer les transformations

1. Inra, UMR Métafort, Equipe TSE

63122 Saint Genès Champanelle, France

2. Convênio Embrapa-Cirad, Tv Enéas Pinheiro s/n, CEP 66095-100, Belém-PA, Brésil

* Auteur pour la correspondance

Tél. : +33 (0)4 73624641 ; fax : +33 (0)4 73624645

E-mail : nhostiou@clermont.inra.fr durables de ces élevages. Les troupeaux, pour la production de lait et de veaux, sont conduits toute l'année au pâturage avec des chargements de moins de $1 \mathrm{UA}^{1}$ par hectare par an (17). Les systèmes d'élevage se caractérisent par de faibles niveaux d'équipements, de mécanisation et d'intrants (2). Ils sont peu productifs (4 à 5 litres de lait/vache/jour), grands consommateurs d'espace, et les prairies sont soumises à des processus d'envahissement par la flore adventice (19). Plusieurs facteurs explicatifs sont mis en avant: des fonctions de l'élevage autres que productives (accumulation, spéculation foncière), le peu d'expérience des éleveurs, leurs faibles capacités monétaires, un appui technique peu structuré $(8,13)$.

Des études mettent en avant une autre contrainte à la transformation et à l'intensification des élevages amazoniens : le travail $(9$, 17). En effet, les collectifs de travail reposent essentiellement sur

\footnotetext{
${ }^{1}$ Unité animale (correspond à une vache de $450 \mathrm{~kg}$ de poids vif)
} 
la main-d'œuvre familiale avec peu de salariat $(16,18)$ et avec des compositions différentes entre les fermes : le collectif de travail peut être composé du seul chef d'exploitation ou de plusieurs groupes familiaux résidant sur la ferme (1). Dans bien des cas, la force de travail n'est pas jugée suffisante pour mener à bien les tâches dans ces systèmes herbagers, car les charges en travail s'avèrent lourdes, difficiles à gérer et exigeantes en main-d'œuvre (16). L'emploi d'ouvriers est limité par les faibles capacités monétaires des familles (19). De nombreuses activités sont réalisées manuellement (traite, coupe de la végétation adventice dans les prairies, implantation de prairies). En outre, les élevages sont marqués par l'agrandissement des structures (surface herbagère et troupeau bovin) augmentant les charges en travail au fil des années (8). La cespondance entre la force de travail disponible et les activités d'élevage à réaliser induirait les processus d'envahissement des prairies ou encore l'arrêt de la production laitière observé dans certaines fermes (11).

De ce fait, l'organisation du travail constituerait un frein aux processus d'intensification des élevages et au développement durable de la production laitière. Cependant le travail n'est quasiment pas abordé dans la littérature scientifique dans le cas des élevages amazoniens et aucune étude privilégiant une approche technique n'a jusqu'à présent été menée sur ce thème. Les données disponibles restent d'ordre général et se réfèrent au nombre de travailleurs et à la composition de la force de travail (répartition entre la maind'œuvre familiale, temporaire et permanente) (19).

L'objectif de cette étude a été de contribuer à mieux connaitre l'organisation du travail dans des fermes «lait-viande» en termes de temps, de volumes d'activités et de stratégies d'éleveurs, à partir d'une analyse de sept exploitations lait-viande sur le front pionnier de la route Transamazonienne. Il s'agit de mettre en évidence que le travail est une composante essentielle à prendre à compte par les acteurs de la recherche-développement.

\section{MATERIEL ET METHODES}

\section{Zone d'étude}

L'étude a été réalisée auprès d'éleveurs de bovins lait-viande de la commune d'Uruará sur le front pionnier de la Transamazonienne dans l'Etat du Pará au Brésil, de 2000 à 2003, lors d'un travail de doctorat (11) (figure 1). Cette région a fait l'objet d'un plan de colonisation agricole dans les années 1970 suite à l'ouverture de la route. Les exploitations agricoles se mettent en place le long de la Transamazonienne et des chemins vicinaux perpendiculaires à cet axe principal. Actuellement, la population est estimée à près de 45000 habitants dont 70 p. 100 établis en milieu rural (12). Avec près de 3000 familles paysannes et 70 p. 100 des fermes de moins de 150 ha, l'agriculture est essentiellement le fait de petits propriétaires ruraux (20).

\section{Méthode «bilan travail »}

Des informations ont été collectées sur l'organisation du travail lors d'un suivi annuel des pratiques de conduite du troupeau et d'utilisation des prairies auprès de sept éleveurs (11). Les sept fermes ont été sélectionnées à partir des résultats d'une typologie sur les structures de production de façon à couvrir les principaux groupes identifiés (tableau I). Du fait de la taille de l'échantillon, les résultats n'ont pas vocation à être généralisés, l'approche privilégiant la compréhension des réalités de terrain.

Les données recueillies portaient sur l'organisation de la maind'œuvre (par qui sont réalisées les activités) et sur les temps consacrés aux différentes tâches (lait, troupeau, prairies). La méthode du bilan travail (5) a été utilisée pour l'analyse. Elle a été développée par l'Inra et l'Institut de l'élevage pour répondre à une préoccupation croissante sur l'organisation et les conditions de travail dans des élevages français : elle a pour objectif d'intégrer la dimension travail dans l'analyse du fonctionnement des systèmes d'élevage (6), et vise à quantifier le travail relatif à la conduite du troupeau et des surfaces sur une campagne annuelle (7). Elle repose sur plusieurs principes.

Tous les travailleurs ne sont pas équivalents, et sont distinguées (5) :

- la cellule de base, composée des travailleurs permanents pour lesquels l'activité d'élevage est prépondérante en temps comme en revenu ;

- la main-d'œuvre hors cellule de base regroupant les bénévoles, l'entraide et l'intervention de salariés temporaires.

Les diverses tâches sont différenciées selon leur rythme de réalisation :

- le travail d'astreinte s'effectue quotidiennement, et il est difficile à concentrer et surtout à différer. Pour l'élevage, il correspond aux soins journaliers apportés aux animaux (surveillance, alimentation, assistance des mises bas, etc.). Il est quantifié en heures par jour ;
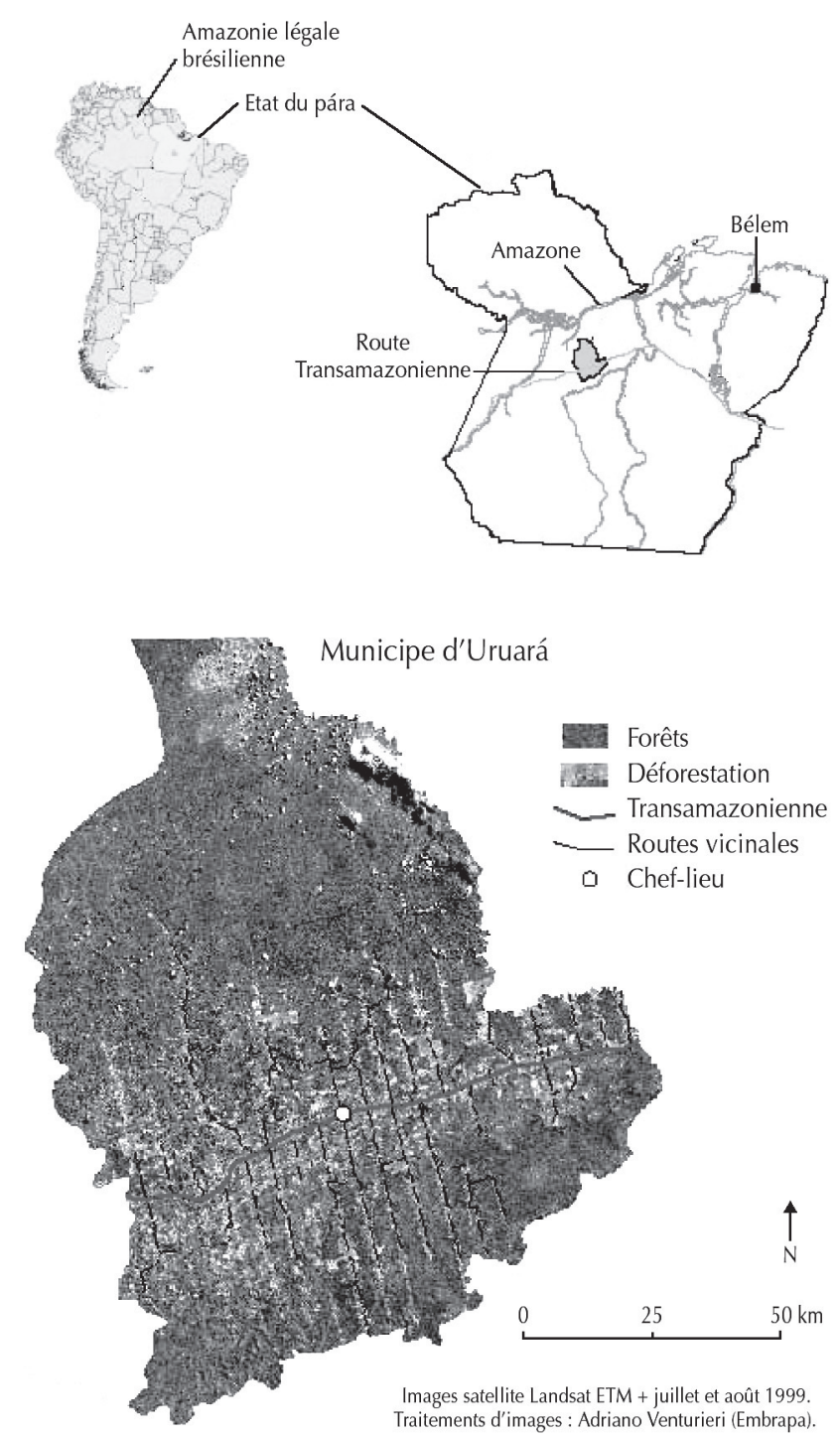

Figure 1 : localisation du municipe d'Uruará. 
Tableau I

Principales caractéristiques des sept exploitations lait et viande

\begin{tabular}{|c|c|c|c|c|c|c|}
\hline Ferme & Type de systèmes de production & $\begin{array}{l}\text { Surface } \\
\text { totale (ha) }\end{array}$ & $\begin{array}{l}\text { Surface en } \\
\text { herbe (ha) }\end{array}$ & $\begin{array}{l}\text { Effectif } \\
\text { bovin }\end{array}$ & $\begin{array}{c}\text { Effectif vaches } \\
\text { pour le lait }\end{array}$ & $\begin{array}{l}\text { Production } \\
\text { lait (I/an) }\end{array}$ \\
\hline Boa & Mixte lait-veaux & 100 & 53 & 31 & 11 & 5300 \\
\hline Cus & & 100 & 70 & 80 & 33 & 15500 \\
\hline Dav & & 105 & 75 & 120 & 45 & 21800 \\
\hline Dar & Intensif lait & 20 & 16 & 38 & 17 & 17200 \\
\hline Dan & Diversifié élevage/culture & 100 & 32 & 47 & 13 & 6000 \\
\hline Don & & 100 & 90 & 74 & 14 & 8800 \\
\hline Iri & Mixte lait-veaux + engraissement & 100 & 80 & 157 & 70 & 51700 \\
\hline
\end{tabular}

- le travail de saison réunit les tâches plus faciles à différer ou à concentrer, qu'il s'agisse des cultures, des fourrages ou des troupeaux (manipulations périodiques par exemple). Il est quantifié en nombre de jours par an.

Le temps disponible calculé représente le temps qui reste à la cellule de base pour réaliser les activités non quantifiées, autrement dit sa marge de manœuvre. Il est exprimé en heures par an et est utilisé comme un élément de diagnostic. Il est calculé par période en déduisant les dimanches, le travail d'astreinte, le travail rendu et les journées de travaux saisonniers.

Les stratégies d'éleveurs face au travail ont été identifiées en regroupant les cas présentant des similarités quant aux volumes horaires (travail d'astreinte, travail de saison, temps disponible calculé) et les déroulés calendaires en lien avec les pratiques de conduite des troupeaux et d'utilisation des prairies.

\section{RESULTATS}

\section{Caractéristiques structurelles des exploitations lait-viande}

Dans les sept fermes lait-viande, le pâturage représentait la première forme de mise en valeur agricole des terres déboisées avec des surfaces de 16 à 90 ha (tableau I). Les prairies étaient installées sur défriche forestière ou recrû ligneux suite à la coupe des arbres et au brûlis. L'espèce fourragère prédominante était Brachiaria brizantha. La surface en herbe était découpée physiquement par des clôtures délimitant des parcelles. Les troupeaux bovins ont été conduits au pâturage toute l'année. L'herbe a composé la base exclusive de leur alimentation. Les bovins étaient issus de croisements entre des races taurines (Hollandaise) et zébus (Nelore, Gir, IndoBrasil, etc.). Les effectifs allaient de 31 à 157 têtes dont 11 à 70 vaches destinées à la production mixte lait-veau. La quantité de lait produite par exploitation a varié de 5300 à 517001 sur l'année.

\section{Composition de la cellule de base}

Les exploitations se différenciaient par le nombre de personnes de la cellule de base et la composition du collectif de travail (tableau II). Dans cinq fermes, la cellule de base reposait sur la main-d'œuvre familiale. Dans deux élevages, l'emploi de salariés permanents avait fait suite à la sortie progressive de la main-d'œuvre familiale (femme, enfants) pour s'occuper d'un grand troupeau (Iri) ou pour aider un producteur suite à la mise en place d'une activité de transformation du lait (Dar). Dans quasiment toutes les fermes, le propriétaire de la terre composait la cellule de base. Dans une ferme (Don), un des fils assurait les activités d'élevage, son père (le propriétaire de la terre) ne prenant pas part aux travaux d'élevage. La cellule de base pouvait compter, en règle générale, sur l'aide bénévole des autres membres de la famille. Cependant, la participation de la famille est apparue fortement liée à la structuration du groupe familial et à l'importance des activités agricoles dans la formation de ses revenus. Ainsi, un éleveur (Boa) travaillait seul : sa famille résidait en ville et ses enfants avaient leur propre situation professionnelle. Dans la ferme de Don, la cellule de base a pu compter sur l'aide des différents membres de sa famille. Cette forte implication était en partie liée à l'organisation de la famille, plusieurs unités domestiques résidant sur l'exploitation parentale.

\section{Travail d'astreinte avec l'atelier laitier}

Dans ces fermes, le travail d'astreinte correspondait aux soins journaliers avec le troupeau (traite, surveillance, soins et rassemblement des vaches en production et veaux) auxquels s'est ajoutée la commercialisation du lait du fait de son rythme quotidien et difficile à différer. Sur l'année, le volume de travail d'astreinte, assuré par la cellule de base, a varié de 1280 à 3955 h (tableau III). De forts écarts journaliers ont été notés car le nombre d'heures a varié de 2 h à 6 h 30 par personne de la cellule de base. Plusieurs facteurs semblaient expliquer cette variabilité : le nombre de membres de la cellule de base, l'effectif des femelles à traire, la quantité de lait et le mode de commercialisation. La traite, réalisée manuellement

\section{Tableau II}

Composition de la cellule de base

\begin{tabular}{llll} 
Ferme & $\begin{array}{l}\text { Nb. de } \\
\text { personnes }\end{array}$ & Composition & $\begin{array}{l}\text { Aide bénévole } \\
\text { de la famille }\end{array}$ \\
\hline Boa & 1 & Producteur & $/$ \\
Don & 1 & Fils & Père, neveux, frères \\
Cus & 1 & Producteur & Femme et enfants \\
Dav & 2 & Producteur + femme & Petit-fils \\
Dan & 2 & Producteur + fils & Femme et fils \\
Dar & 2 & Producteur + salarié & $/$ \\
Iri & 3 & Producteur + salariés & Femme \\
\hline
\end{tabular}

* Personnes non rétribuées monétairement pour leur travail 
Tableau III

Volumes d'activités pour le travail d'astreinte et le travail de saison

\begin{tabular}{|c|c|c|c|c|c|c|c|c|c|c|}
\hline \multirow[t]{2}{*}{ Ferme } & \multirow{2}{*}{$\begin{array}{l}\text { Nb. de } \\
\text { vaches }\end{array}$} & \multirow{2}{*}{$\begin{array}{l}\text { Surface en } \\
\text { herbe (ha) }\end{array}$} & \multirow[t]{2}{*}{ CB } & \multicolumn{2}{|c|}{ Travail d'astreinte } & \multicolumn{4}{|c|}{ Travail de saison } & \multirow[t]{2}{*}{ TDC/PCB } \\
\hline & & & & h/an & $h / j / P C B$ & Troupeaux & Prairies & Territoire & $\begin{array}{l}\text { \% assuré } \\
\text { par le CB }\end{array}$ & \\
\hline Boa & 11 & 53 & 1 & 2250 & 6 h 30 & 0 & 17 & 0 & 100 & 444 \\
\hline Don & 14 & 90 & 1 & 1580 & $4 \mathrm{~h} 30$ & 56 & 37 & 0 & 53 & 920 \\
\hline Dav & 45 & 70 & 2 & 2070 & 2 h 45 & 56 & 176 & 96 & 44 & 1265 \\
\hline Cus & 33 & 70 & 1 & 2130 & 6 h 00 & 21 & 96 & 0 & 44 & 522 \\
\hline Dan & 12 & 32 & 2 & 1615 & 2 h 00 & 50 & 144 & 81 & 91 & 1128 \\
\hline Dar & 17 & 16 & 1 & 1280 & 1 h 45 & 4 & 29 & 4 & 95 & 1848 \\
\hline Iri & 70 & 80 & 3 & 3955 & $3 \mathrm{~h} 30$ & 98 & 154 & 0 & 66 & 1160 \\
\hline
\end{tabular}

CB : cellule de base ; PCB : personne de la cellule de base

TDC : temps disponible calculé

une fois par jour, a été la première activité en volume de temps dans toutes les fermes ( 2 h 20 en moyenne par ferme par jour), avec cependant de forts écarts ( $50 \mathrm{~min}$ à $3 \mathrm{~h}$ ). La durée journalière dédiée à cette activité a été corrélée à l'effectif des vaches à traire. Du fait d'un marché du lait informel et sans industrie de transformation, la commercialisation était réalisée par les producteurs eux-mêmes, après la traite. Ils y consacraient en moyenne $2 \mathrm{~h}$ par jour, avec des écarts de 30 min à $3 \mathrm{~h}$. Le plus faible volume horaire journalier a été observé dans une ferme (Dar) où l'éleveur vendait son lait à un seul point de vente, alors que le plus élevé ( 6 h 30) correspondait à un éleveur commercialisant son lait à vélo auprès d'un réseau de clientèle urbaine.

\section{Travail de saison}

Dans cette étude, le travail de saison a compris les tâches avec le troupeau bovin, les prairies cultivées et le territoire. Sur l'année, le travail de saison total a été de 17 à 328 journées de travail. Selon les exploitations, la cellule de base a mobilisé de la main-d'œuvre externe (aide bénévole de la famille et/ou ouvriers) à des degrés divers. Les principaux résultats concernant le travail de saison sont indiqués dans le tableau III.

Le travail de saison avec le troupeau bovin a regroupé la distribution de la complémentation minérale, les opérations de prophylaxie (vaccination), les soins et la surveillance du bétail en pension. Il a été assuré principalement par la cellule de base qui pouvait avoir recours à de l'aide familiale. Le volume d'activités a varié de 0 à 98 jours, en fonction notamment de l'effectif bovin. Un travail de saison nul a été observé dans une ferme où les activités étaient similaires à du travail d'astreinte.

Les travaux de saison liés à la gestion des prairies ont regroupé le sarclage manuel de la végétation adventice, l'arrachage du système racinaire de mauvaises herbes, l'application d'herbicides, la réparation et pose de clôtures, le semis de la graminée fourragère lors des processus de restauration de prairies. Toutes ces activités ont été réalisées manuellement. Dans ces élevages herbagers, où le troupeau paissait l'herbe toute l'année, de forts écarts ont été enregistrés (17 à 176 jours par an). La surface en herbe n'est pas apparue être un facteur de différenciation du volume de travail de saison entre les fermes. Les différences ont semblé plutôt liées aux activités réalisées et aux décisions et pratiques des éleveurs pour entretenir leur surface herbagère. Des activités ont été déléguées à de la main-d'œuvre salariée ou bénévole. Les activités pour lutter contre l'envahissement des prairies ont été les plus consommatrices en temps. Le sarclage de la flore adventice (60 jours de travail en moyenne par élevage), qui consiste à couper la partie aérienne des adventices avec une houe ou une faucille, a été réalisé dans toutes les fermes avec de grandes disparités (13 à 124 jours). L'arrachage, qui consiste à éliminer la plante adventice avec son système racinaire après la coupe de la partie aérienne, et l'application d'herbicides ont été réalisés dans trois fermes (2 à 30 jours). Des activités ont été déléguées à de la main-d'œuvre salariée ou bénévole.

Le travail de saison pour la gestion du territoire a regroupé les opérations d'implantation de nouvelles surfaces herbagères sur la forêt : coupe des arbres, brûlis et semis de la graminée fourragère. Enregistré dans trois fermes, les éleveurs y ont consacré 4 à 96 jours.

\section{Temps disponible calculé}

Le temps disponible calculé a été compris entre 444 et 1848 heures par an et par personne de la cellule de base, exprimant des différences considérables quant aux marges de manœuvre dont disposaient les éleveurs pour réaliser d'autres activités pendant l'année (avec les cultures par exemple). Il existait un lien entre le temps disponible calculé et le nombre de personnes de la cellule de base : il a été plus élevé quand la cellule de base était composée de 2 ou 3 membres. Il était par contre plus faible quand le volume de travail d'astreinte était élevé par personne de la cellule de base (tableau III).

\section{Stratégies d'éleveurs et organisation du travail sur une année}

Les éleveurs ont eu recours à trois stratégies pour tenir compte des contraintes de travail dans les objectifs de conduite du troupeau et d'utilisation des prairies (tableau IV).

\section{Simplifier le travail et la conduite toute l'année (type A)}

L'organisation du travail a été marquée par une période creuse sur plusieurs mois. Pour se libérer du temps de travail, les cellules de base (1 personne) ont joué sur le temps consacré au travail d'astreinte par l'arrêt de la traite et/ou en modifiant le mode de commercialisation. Les éleveurs ont cherché à libérer du temps 
Tableau IV

Stratégies d'éleveurs

\begin{tabular}{|c|c|c|c|c|c|c|}
\hline $\begin{array}{l}\text { Type de } \\
\text { stratégies }\end{array}$ & Ferme & $\begin{array}{l}\text { Travail } \\
\text { d'astreinte } \\
\text { (TA) }\end{array}$ & $\begin{array}{l}\text { Périodes de } \\
\text { pointe avec du } \\
\text { travail de saison }\end{array}$ & $\begin{array}{l}\text { Temps disponible } \\
\text { calculé (TDC) }\end{array}$ & $\begin{array}{l}\text { Pratiques } \\
\text { et objectifs }\end{array}$ & $\begin{array}{l}\text { Collectifs de travail } \\
\text { (CB : cellule de base) }\end{array}$ \\
\hline \multirow[t]{2}{*}{ A } & Boa & \multirow{2}{*}{$\begin{array}{l}\text { Période avec } \\
\text { un TA très réduit }\end{array}$} & \multirow[t]{2}{*}{ Non } & \multirow{2}{*}{$\begin{array}{l}\text { TDC annuel faible } \\
\text { mais très évolutif } \\
\text { pendant l'année }\end{array}$} & \multirow{2}{*}{$\begin{array}{l}\text { Faciliter la conduite } \\
\text { des vaches toute } \\
\text { l'année (une parcelle } \\
\text { pour les vaches, } \\
\text { utilisation continue) }\end{array}$} & CB (éleveur) \\
\hline & Don & & & & & $\begin{array}{l}\text { CB (éleveur) + } \\
\text { aide familiale }\end{array}$ \\
\hline \multirow[t]{2}{*}{ B } & $\begin{array}{l}\text { Dav } \\
\text { Cus }\end{array}$ & \multirow{2}{*}{$\begin{array}{l}\text { TA fonction de } \\
\text { la répartition des } \\
\text { vêlages (pic en } \\
\text { saison sèche) et } \\
\text { de l'état des routes } \\
\text { (difficultés en saison } \\
\text { des pluies) }\end{array}$} & \multirow[t]{2}{*}{$\begin{array}{l}\text { En saison des } \\
\text { pluies et fin } \\
\text { de saison sèche }\end{array}$} & \multirow[t]{2}{*}{$\begin{array}{l}\text { TDC évoluant } \\
\text { selon les périodes }\end{array}$} & \multirow{2}{*}{$\begin{array}{l}\text { Simplifier la conduite } \\
\text { pendant les périodes } \\
\text { de pointes } \\
\text { (regroupement des } \\
\text { lots, mise en défens } \\
\text { de parcelles) }\end{array}$} & $\begin{array}{l}\mathrm{CB}+\text { aide familiale }+ \\
\text { salariat pour } \\
\text { les prairies }\end{array}$ \\
\hline & Dan & & & & & $\mathrm{CB}+$ aide familiale \\
\hline \multirow[t]{2}{*}{$\mathrm{C}$} & Dar & \multirow[t]{2}{*}{ Stable } & \multirow[t]{2}{*}{ Non } & \multirow[t]{2}{*}{ Stable } & \multirow{2}{*}{$\begin{array}{l}\text { Privilégier la } \\
\text { productivité laitière } \\
\text { et des prairies toute } \\
\text { l'année (rotation rapide, } \\
\text { sarclage manuel) }\end{array}$} & \multirow{2}{*}{ Salariat } \\
\hline & Iri & & & & & \\
\hline
\end{tabular}

pour mener à bien d'autres activités agricoles non liées à l'élevage. Toute l'année, les éleveurs ont cherché à simplifier la conduite des bovins en allouant les parcelles proches de l'étable aux laitières, et par l'utilisation continue d'une parcelle ou d'un bloc de parcelles. Le travail de saison a été assuré par la cellule de base, avec l'aide de la famille si celle-ci résidait sur la ferme. Les activités avec les prairies ont été étalées sur plusieurs mois de façon à répartir les besoins en main-d'œuvre et à ne pas recourir au salariat.

\section{Adapter la conduite lors des périodes de pointe en travail (type B)}

Des élevages ont été caractérisés par des alternances de périodes de travail plus ou moins intenses. Les volumes horaires pour l'atelier laitier ont évolué en fonction de la répartition des vêlages (pic en saison sèche) et de l'état des routes (difficultés de circulation en saison des pluies). La cellule de base a pu recourir à l'aide bénévole de la famille pour la traite lors des périodes de vêlages. Des concurrences ont été observées entre les travaux quotidiens avec le lait et les activités saisonnières avec les prairies. En saison des pluies, les cellules de base ont réalisé le sarclage manuel des prairies. En fin de saison sèche, elles se sont chargées des activités d'implantation de nouvelles prairies sur la forêt (coupe des arbres, semis), de la pose de clôtures. Pour mener à bien ces tâches, elles ont eu recours à de la main-d'œuvre externe familiale ou salariée. Lors des périodes en travail plus mouvementées, les pratiques ont été modifiées pour réduire le temps consacré à la conduite du troupeau (regroupement ou séparation des lots, utilisation continue d'une parcelle, regroupement de parcelles, etc.).

\section{Employer de la main-d'œuvre et répartir les activités au sein des collectifs (type $C$ )}

Dans des élevages, la conduite a été de chercher à maintenir des niveaux de productivité laitière élevés et des prairies en état toute l'année (rotation rapide des vaches sur les parcelles, sarclage manuel des prairies). Le travail d'astreinte et le temps disponible calculé se sont caractérisés par leur stabilité pendant l'année. Cette organisation a été liée à plusieurs facteurs :

- le salariat à temps plein ;

- la répartition des activités saisonnières pendant la campagne annuelle de façon à limiter les périodes de pointe et l'emploi d'ouvriers agricoles payés à la tâche ou à la journée ;

- l'étalement des vêlages sur l'année ;

- la répartition des tâches entre les travailleurs ; par exemple dans une ferme, le salarié se consacrait à la traite et l'éleveur à la vente; dans une autre, l'exploitant réalisait les travaux quotidiens et les salariés les travaux de saison.

\section{DISCUSSION}

\section{Organisation du travail dans les fermes lait-viande}

Cette étude exploratoire, en s'appuyant sur la méthode bilan travail, est une première analyse de l'organisation technique du travail dans des élevages familiaux lait-viande en frontière agricole amazonienne. Du fait de la taille de l'échantillon, les résultats n'ont pas vocation à être généralisés ; cependant cette étude apporte des éléments pour caractériser la composition des collectifs de travail, les temps de travaux quotidiens et saisonniers, la répartition des tâches entre les travailleurs de la cellule de base et la maind'œuvre externe, et des facteurs explicatifs.

Les collectifs de travail comprenaient un à trois membres par cellule de base, qui pouvaient compter sur l'aide familiale selon leur niveau d'implication, notamment celui des enfants, dans l'activité d'élevage. De fortes variations sont apparues entre les fermes en termes de volumes d'activités pour les activités quotidiennes de l'atelier lait, et les travaux de saison avec les prairies et les troupeaux. La production laitière a induit des activités journalières communes à toutes les exploitations, dont les plus consommatrices 
en temps ont été la traite et la vente du lait du fait des pratiques (traite manuelle) et de l'organisation actuelle de la filière (vente informelle). Ces deux tâches ont été caractérisées par de fortes variabilités des quantités de travail. Le facteur explicatif principal pour la traite a été le nombre de travailleurs et l'effectif de vaches à traire, et pour la vente du lait l'organisation du réseau de clientèle. La cellule de base a réalisé le travail d'astreinte, avec dans certains cas une aide de la famille lors des périodes en travail plus intenses (pic de vêlages par exemple). Les travaux de saison ont été identifiés avec les troupeaux bovins, les prairies cultivées et le territoire. Ces activités, réalisées manuellement, étaient exigeantes en force de travail. La cellule de base a compté sur sa propre capacité de travail ou a eu recours à de la main-d'œuvre externe souvent salariée pour des activités d'entretien des prairies ou de coupe des arbres.

\section{Propositions d'actions de recherche-développement sur le thème du travail}

Le travail est un facteur à prendre en compte pour appuyer la transformation des pratiques des éleveurs et l'organisation technique des systèmes d'élevage, ceci pour garantir leur durabilité économique, écologique et sociale. Des solutions doivent être proposées à ces éleveurs :

- pour améliorer la conduite de l'élevage et le travail pendant l'année car les éleveurs doivent gérer de nombreuses tâches (quotidiennes et saisonnières) avec les troupeaux, les prairies (et parfois les cultures) en fonction de la main-d'œuvre ;

- pour faire face à des modifications de la main-d'œuvre familiale (départ des enfants) ou encore à des redimensionnements de l'atelier d'élevage (agrandissement du troupeau et des surfaces en herbe), et des activités agricoles (diversification des productions).

Les recherches auront pour objectif de mieux comprendre l'organisation du travail pour identifier les marges de progrès et les solutions possibles. Plusieurs pistes sont proposées. Il s'agit de poursuivre les études sur les temps de travaux auprès d'un plus large échantillon d'élevages afin d'élaborer des références sur ces systèmes d'élevage (3). Des critères semblent pertinents pour sélectionner les exploitations : la composition des collectifs de travail, les combinaisons d'activités agricoles (élevage, cultures) et non agricoles, le mode de commercialisation du lait. Cette étude a mis en évidence des problèmes de durées de travail, de gestion des périodes de pointe et de pénibilité de tâches, mais elle ne les a certainement pas tous identifiés (10). Les préoccupations et les attentes des éleveurs vis-à-vis de leur travail sont à prendre en considération pour orienter les actions de recherche-développement. D'après les résultats, un objectif prioritaire viserait à alléger les charges en travail (quotidiennes et saisonnières) ainsi qu'à réduire la pénibilité physique de certaines tâches. Les solutions pourraient porter sur les équipements/bâtiments (investissement dans des salles et du matériel de traite, accès à la mécanisation pour l'entretien des prairies), la conduite des troupeaux (répartir les vêlages). Il est également nécessaire de réfléchir à la main-d'œuvre (faciliter le recours à l'emploi d'ouvriers) et à l'organisation de la filière lait (mettre en place une laiterie). Les propositions et les messages doivent tenir compte des différentes stratégies d'éleveurs pour organiser leur travail et l'intégrer dans leurs objectifs techniques : réduire le travail, limiter les périodes de pointe et/ou recomposer la main-d'œuvre de l'exploitation (4). L'aspect économique (gestion de la trésorerie et revenus) doit d'être pris en compte afin de proposer des solutions adaptées aux capacités d'investissements des élevages.

\section{CONCLUSION}

Ce travail a été nécessaire pour permettre de poursuivre les réflexions sur la durabilité des systèmes d'élevage amazoniens. Des actions de recherche-développement sur le thème du travail devraient donc être mises en place en partenariat avec les agriculteurs de façon à lever des freins pour l'adoption de techniques de conduite plus rationnelles des élevages et la transformation des pratiques des éleveurs. Il s'agit également d'améliorer les conditions de travail et de vie des agriculteurs pour conforter la transmissibilité et la «vivabilité » des exploitations familiales. Les travaux pluridisciplinaires ont un rôle majeur à jouer de manière à associer plusieurs points de vue et éclairer ainsi les différentes dimensions des exploitations, qu'elles soient techniques, sociales ou encore économiques.

\section{Remerciements}

Les auteurs remercient l'Embrapa Amazonie orientale et le Cirad, département Emvt, pour leurs collaborations, ainsi que B. Dedieu (Inra-SAD, UMR Métafort, équipe transformations des systèmes d'élevage) pour ses relectures.

\section{BIBLIOGRAPHIE}

1. ALVES A.M.N., 2004. Dynamique et reproduction sociale de I'agriculture familiale des fronts pionniers d'Amazonie brésilienne. Exemple du paysannat de la Transamazonienne associant élevage laitier et agriculture. Mémoire DEA, Ecole des hautes études en sciences sociales, Paris, France.

2. AZEVEDO DE P.C., CARVALHO DE R.A. TEIXEIRA R.N.G., SARMENTO C.M.B., FILHO J.A.R., GONCALVES C.A., OLIVEIRA R.P., 1994. Caracteristicas dos sistemas de produção de gado de corte na Região Bragantina. Belém, Brésil, Embrapa-Captu, 35 p. (Coll. Documents $n^{\circ} 79$ )

3. Chauvat S., SEEGers J., N'GuYEN B., CleMENT B., 2003. Le travail d'astreinte en élevage bovin laitier. Synthèse nationale d'enquêtes bilan-travail. Analyse des aspirations et des solutions envisagées par les éleveurs du dispositif Réseaux d'élevage. Paris, France, Institut de l'élevage, $51 \mathrm{p}$.

4. CITTADINI R., DEDIEU B., DERAIL L., PEREZ R., 2001. Trabajo y tecnología en explotaciones ganaderas de la provincia de Buenos Aires. In: Neiman G. Ed., Trabajo de campo. Produccion, technologia y empleo en el medio rural. Buenos Aires, Argentina, CICCUS, p. 120-133.

5. DEDIEU B., CHAUVAT S., SERVIERE G., TCHAKERIAN E., 2000. Bilan travail pour l'étude du fonctionnement des exploitations d'élevage, méthodes d'analyse. Paris, France, Institut de l'élevage / Inra, 27 p. (Coll. Lignes)

6. DEDIEU B., SERVIERE G., 2001. Organisation du travail et fonctionnement

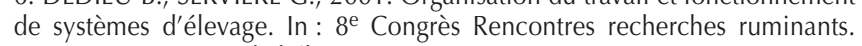
Paris, France, Institut de l'élevage / Inra, p. 245-250.

7. DEDIEU B., SERVIERE G., 1999. Caractériser et évaluer I'organisation du travail en élevage. La méthode Bilan Travail. Fascade, 1 : 1-4.

8. FERREIRA L.A., 2001. Le rôle de l'élevage bovin dans la viabilité agroécologique et socio-économique des systèmes d'exploitations agricoles familiaux en Amazonie orientale brésilienne - le cas d'Uruará. Thèse Doct., INA-PG, Paris, France, 187 p. 
9. FERREIRA L.A., TOURRAND J.F., VEIGA J.B., QUANZ D., 1995 Produção leiteira na área de fronteira agrícola na Amazônia - o caso de Altamira. Belém, Brésil, Embrapa, 53 p.

10. GUILLAUMIN A., KLING-EVEILLARD F., MOREAU J.C., SEEGERS J., 2005. Résultats d'enquêtes en Aquitaine. Quand les éleveurs laitiers parlent de leurs conditions de travail. Trav. Innovations, 115 : 30-35.

11. HOSTIOU N., 2003. Pratiques et stratégies de gestion des ressources herbagères cultivées par des éleveurs laitiers sur un front pionnier en Amazonie brésilienne : le cas du municipe de Uruará. Thèse Doct., INAPG, Paris, France, 206 p.

12. IBGE, 2000. Censo demógrafico 2000. Resultados do Universo População residente, por situação do domicílio e sexo, segundo as grandes regiões e as Unidades da Federação - Brasil - grandes regiões. http://www.ibge.gov.br

13. MUCHAGATA M., BROWN K., 2003. Cows, colonists and trees: rethinking cattle and environmental degradation. Agric. Syst., 76: 797-816.

14. POCCARD-CHAPUIS R., 2004. Les réseaux de la conquête. Rôle des filières bovines dans la structuration de l'espace sur les fronts pionniers d'Amazonie orientale brésilienne. Thèse Doct. Géographie, université Paris X, Nanterre, France, $435 \mathrm{p}$

15. POCCARD-CHAPUIS R., PIKETTY M.G., VEIGA J.B., HOSTIOU N., TOURRAND J.F., 2002. Milk production, regional development and sustainability in the Eastern Brazilian Amazon. In : Actes colloque Les systèmes agroalimentaires localisés : produits, entreprises et dynamiques locales, Montpellier, France, 16-18 oct. 2002. Montpellier, France, Ciradtera, 12 p., CD-Rom.
16. TOPALL O., 1995. L'arbre et I'herbe en zone tropicale humide. Gestion des pâturages sur une frontière agricole amazonienne dans la région de Marabá au sud de l'état du Pará, Brésil. In : Pichot J., Sibelet N., Lacoeuilhe J.J., éds, Actes séminaire Fertilité du milieu et stratégies paysannes sous les tropiques humides, Montpellier, France, 13-17 nov. 1995. Montpellier, France, Cirad-sar, p. 260-265.

17. TOURRAND J.F., VEIGA J.B., QUANZ D., FERREIRA L.A., SIMAO NETO M., 1998. Produção leiteira em área de fronteira agrícola na Amazônia - o caso do município de Uruará. In: Homma A.K.O. Ed. Amazônia : meio amabiente e desenvolvimento agrícola. Bélem, Brésil, Embrapa-SPI, p. 345-365

18. VEIGA J.B., POCCARD-CHAPUIS R., PIKETTY M.G., TOURRAND J.F., 2001. Produção leiteira e o desenvolvimento regional na Amazônia oriental. Belém, Brésil, Embrapa, 24 p. (Coll. Documentos n ${ }^{80}$ )

19. VEIGA J.B., TOURRAND J.F., 2000. Sinteses das limitações e potencialidades da produção leiteira na Amazônia oriental. In Veiga J.B. Tourrand J.F., Eds, Produção leiteira na Amazônia oriental, situação atual e persectivas. Belém, Brésil, Embrapa Amazônia oriental, p. 227-234.

20. VEIGA J.B., TOURRAND J.F., QUANZ D., 1996. A pecuária na fronteira agrícola da Amazônia: o caso do município de Uruará Pa, na Transamazônica. Belém, Brésil, Embrapa-Cpatu, 61 p. (Coll. Documentos

Reçu le 09.02.2005, accepté le 17.02.2006

\section{Summary}

Hostiou N., Tourrand J.F., Veiga J.B. Work Organization in Dairy and Beef Family Farms on an Amazonian Pioneer Front in Brazil. Study from Seven Work Assessment Surveys

This study puts under a new light dairy family farms on the Amazonian frontier in Brazil: the technical organization of work. Labor appears to be an essential factor of livestock management and a constraint to the sustainable development of the farms. But, very little knowledge has been gathered to date. To show the work organization over one year, the working time was quantified and working groups described based on the so-called Travail Bilan method in seven dairy/beef farms. The same routine tasks were carried out with the dairy cattle in all the farms, with variations in daily working hours from $1 \mathrm{~h} 45$ to 6 h30 per permanent worker. Differences between farms were related to the number of permanent workers, to the number of milked cows, and to the manner the milk was marketed. During the year, 17 to 328 days were dedicated to seasonal work, including 17 to 176 days to pasture management. Some pasture activities (weeding, sowing, fencing) were delegated to temporary wage earning workers or volunteer family members. Three farmers' strategies were identified based on simplified livestock management or the organization of workers' groups. The study concludes that work is a factor to be taken into account to support the transformation of farming practices and sustainability of Amazonian family farms. Future actions of research-development in the Amazon region will enhance the knowledge necessary to identify possible ways of improvement.

Keywords: Beef cattle - Dairy cattle - Work organization Livestock rearing - Amazonia - Brazil.

\section{Resumen}

Hostiou N., Tourrand J.F., Veiga J.B. Organización del trabajo en las crías familiares de leche y carne en un frente pionero del Amazonas, en Brasil. Estudio a partir de siete encuestas reporte de trabajo

Este estudio privilegió un nuevo enfoque sobre las crías de leche familiares en la frontera agrícola en Brasil: la organización técnica del trabajo. El trabajo parece ser un factor esencial en los comportamientos de cría y un obstáculo a las transformaciones duraderas de las explotaciones. Sin embargo, hasta hoy se han generado pocos conocimientos. Para rendir cuentas de la organización del trabajo a lo largo de un año, los horarios de los trabajos fueron cuantificados y los colectivos de trabajo descritos en siete fincas "leche-carne", a partir del método bilan travail. Las mismas actividades de restricción con el hato lechero fueron realizadas en todas las crías con variaciones en cuanto a los volúmenes de horarios: 1 h4 45 a 6 h30 por día y por persona en la célula de base. Las diferencias entre las fincas estuvieron relacionadas con la composición de la célula de base, con el número de vacas ordeñadas y el modo de comercialización de la leche. En un año, de 17 a 328 días fueron dedicados a las actividades de estación, de los cuáles 17 a 176 días en las praderas cultivadas. Una parte de las actividades en las praderas (arado, plantación, cercas) fueron delegados a la ayuda familiar o a asalariados temporales. Se identificaron tres estrategias de los criadores frente al trabajo, las cuales reposan sobre los comportamientos o la organización de los colectivos de trabajo. El estudio concluye que el trabajo es un factor que debe considerarse para apoyar la transformación de las prácticas de los criadores y la durabilidad de los establecimientos familiares amazónicos. Las futuras acciones de investigacióndesarrollo en el Amazonas permitirán el aumento de los conocimientos para identificar las posibles vías de mejora.

Palabras clave: Ganado bovino - Ganado de carne - Ganado de leche - Organisación del trabajo - Cría de ganado Amazonia - Brasil. 